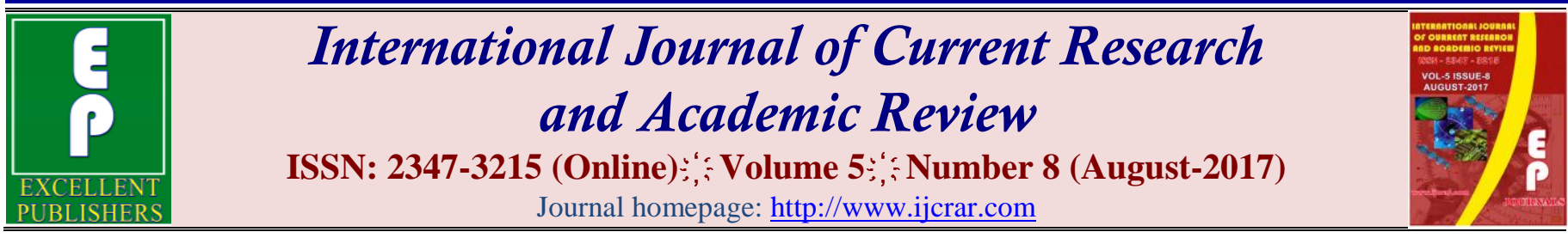

doi: https://doi.org/10.20546/ijcrar.2017.508.004

\title{
Yield and Economics of Irrigated Blackgram under Lay by Method of Pre-Emergence Herbicide Application
}

\author{
C. Agila* and C. R. Chinnamuthu \\ Department of Agronomy, Agricultural College and Research Institute - Madurai, Tamil Nadu, India \\ *Corresponding author
}

\begin{abstract}
Field experiment was conducted during rabi 2015 at Tamil Nadu agricultural university, AC \& RI, Madurai to study yield and economics of irrigated blackgram under lay by method of pre-emergence herbicide application. Results indicated that lay by method were found to superior to individual application with respect to weed control. Among the lay by treatments pre emergence application of pendimethalin at $1.0 \mathrm{~kg} \mathrm{ha}^{-1}$ on 3 DAS followed by hand weeding on 20 DAS and sand mix application of pendimethalin at $1.0 \mathrm{~kg} \mathrm{ha}^{-1}$ immediately after hand weeding significantly reduced the weed growth and recorded higher seed yield $\left(1087 \mathrm{~kg} \mathrm{ha}^{-1}\right)$, net monetary returns (Rs. $40546 \mathrm{ha}^{-1}$ ) and B: C ratio 2.86 . The results significantly superior over rest of the treatments. Uncontrolled weed growth caused reduction in seed yield of blackgram.
\end{abstract}

\section{Article Info}

Accepted: 30 July 2017

Available Online: 20 August 2017

\section{Keywords}

Black gram,

Pre-emergence,

Lay by method,

Yield and economics

\section{Introduction}

Black gram (Vigna mungo L.) is important pulse crop cultivated in worldwide in tropical and sub-tropical regions. India is the largest producer and consumer of pulses accounting for 33.6 per cent of the world area and 24 per cent of the world production of pulses (Pramanik, 2009). Chemical weed management in pulse crops has been found effective and economical (Dungarwal et al., 2003). The crop is not a very good competitor against the weeds and therefore weed control initiatives are essential to ensure proper crop growth, particularly in the early growth period. Application of pre-emergence herbicide last long only a very short period of crop growth. After the loss of herbicide concentration in soil due to various means, the left over weed seeds present in the weed seed bank start to emerge and compete with crops for natural resources. Management of weeds includes cultural, mechanical and chemical besides manual methods.
Though the hand weeding is considered as one of the best methods to check the emergence of weeds throughout the crop duration. Therefore, a field experiment was conducted to study the effect of preemergence herbicides compared with hand weedings and untreated check for evaluating weed control efficacy obtaining high yields of blackgram.

\section{Materials and Methods}

A field experiment was conducted during rabi 2016 at Department of Agronomy, Agricultural College and Research Institute, Tamil Nadu Agricultural University, Madurai respectively $\left(9^{\circ} 54^{\prime} \mathrm{N}\right.$ latitude and $78^{\circ} 54^{\prime} \mathrm{E}$ longitude at an altitude of $147 \mathrm{~m}$ above mean sea level) to study the effect of pre-emergence herbicides on weeds, grain yield of blackgram and the economics involved. The experiment was laid out in randomized block design and replicated thrice. The treatments 
comprised of ten different weed management practices viz., pre-emergence application of pendimethalin at $1 \mathrm{~kg}$ $\mathrm{ha}^{-1}$ alone on 3 DAS or followed by 0.5 or $1.0 \mathrm{~kg} \mathrm{ha}^{-1}$ applied in sequence with or without hand weeding on 20 DAS or quizolofop-ethyl at $0.050 \mathrm{~kg} \mathrm{ha}^{-1}$ on $20 \mathrm{DAS}$. It was compared with hand weeding twice (20 and 40 DAS) weed free check and unweeded check. The soil type of the experimental field are sandy clay loam in texture, neutral in $\mathrm{pH} 7.20$, low Ec $\left(0.46 \mathrm{dSm}^{-1}\right)$, low organic carbon ( 0.29 per cent) medium in available $\mathrm{N}$ (234.26) and in available $\mathrm{P}$ (15.80) and $\mathrm{K}$ content (292.52).The crop was irrigated at critical stages. Need based plant protection measures were given as per the crop protection guide, 2012. The growth attributes were recorded from five selected plants in each plot. Observations on weeds were recorded with the help of a quadrate $(0.5 \mathrm{~m} \times 0.5 \mathrm{~m})$ placed randomly at two places (outside the net plot area) in each treatment at 20,50 DAS and at harvest. The data on weeds were subjected to square root transformation ( $\mathrm{X}+2)$ to normalize their distribution.

\section{Results and Discussion}

\section{Effect on weeds}

The major weeds found in the experimental field were Echinochloa colonum, Echinochola crusgalli and Cynodon dactylon. Among the grasses Cynodon dactylon is the dominant grass weed. Cyperus rotundus and Cyperus iria were the predominant sedges. In sedges Cyperus rotundus is the key sedge weeds in the experimental trial. The important broad leaved weeds were Commelina benghalensis, Cleome viscosa, Convolvulus arvensis, Trianthema portulacastrum, Phyllanthus niruri and Eclipta alba. The weed density and dry weight were recorded at 20, 30 DAS and at harvest (Table 1).

The sequential application of pre-emergence followed by pre-emergence (3 and 20 DAS) with different doses significantly reduced the weed density and weed dry weight compared to other weed control treatments. The pre-emergence application of pendimethalin at $1.0 \mathrm{~kg} \mathrm{ha}^{-}$ ${ }^{1} 3$ DAS $\mathrm{fb}$ HW on 20 DAS + pendimethalin at $1.0 \mathrm{~kg} \mathrm{ha}^{-}$

${ }^{1}$ on $20 \mathrm{DAS} \mathrm{T}_{7}$ recorded low weed density and weed dry weight. The pre-emergence application of pendimethalin at $1.0 \mathrm{~kg} \mathrm{ha}^{-1}$ on $3 \mathrm{DAS} f b$ post-emergence application of quizolofop-ethyl at $0.050 \mathrm{~kg} \mathrm{ha}^{-1}$ on $20 \mathrm{DAS}$ reduced the grass weed density at $50 \mathrm{DAS}$. This might be due to the effect of the post-emergence herbicide over the grassy weeds. The results corroborate with the findings of Dixit et al., (2011). The highest weed dry weight was recorded in unweeded check $T_{10}$. It may be due the undisturbed weed growth during the entire crop growth period.

\section{Effect on crop}

The maximum seed yield was recorded weed free check of $1189 \mathrm{~kg} \mathrm{ha}^{-1}$. Among the herbicides treatments, preemergence application of pendimethalin at $1.0 \mathrm{~kg} \mathrm{ha}^{-1}$ on 3 DAS $f b$ HW on 20 DAS + pendimethalin at $1.0 \mathrm{~kg} \mathrm{ha}^{-1}$ on 20 DAS recorded grain yield of $1087 \mathrm{~kg} \mathrm{ha}^{-1}$ (Table 2 ). The pre-emergence application of pendimethalin at $1.0 \mathrm{~kg} \mathrm{ha}{ }^{-1}$ on 3 DAS $f b$ post-emergence application of quizalofop-ethyl at $0.050 \mathrm{~kg} \mathrm{ha}^{-1}$ on 20 DAS was not effective compared to that of lay by method of preemergence herbicide application which recorded only $782 \mathrm{~kg} \mathrm{ha}^{-1}$ grain yield.

The weed free plot recorded maximum haulm yield of $1803 \mathrm{~kg} \mathrm{ha}^{-1}$. Among the weed control treatments preemergence application of pendimethalin at $1.0 \mathrm{~kg} \mathrm{ha}^{-1}$ on 3 DAS $f b$ HW on 20 DAS + pendimethalin at $1.0 \mathrm{~kg} \mathrm{ha}^{-1}$ on 20 DAS recorded haulm yield of $1729 \mathrm{~kg} \mathrm{ha}^{-1}$. The control plot recorded lowest haulm yield of $1082 \mathrm{~kg} \mathrm{ha}^{-1}$. This result corroborates with the findings of Kalaiselvi et al., (1998).

The highest harvest index ( 0.40 per cent) was recorded with the weed free check. Among the various weed control treatments the pre-emergence application of pendimethalin at $1.0 \mathrm{~kg} \mathrm{ha}^{-1}$ on 3DAS $f b \mathrm{HW}$ on 20 DAS + pendimethalin at $1.0 \mathrm{~kg} \mathrm{ha}^{-1}$ on 20 DAS recorded the higher harvest index of 0.39 per cent. Followed by the pre-emergence application of pendimethalin at $1.0 \mathrm{~kg} \mathrm{ha}^{-1}$ on 3 DAS $f b \mathrm{HW}$ on $20 \mathrm{DAS}+$ pendimethalin at $0.5 \mathrm{~kg}$ $\mathrm{ha}^{-1}$ on 20 DAS registered with harvest index of 0.37 per cent. All other weed control treatments were registered comparable harvest index with each other. The lowest harvest index of 0.31 per cent was observed in the unweeded check.

\section{Economics}

Maximum gross return of Rs. $68111 \mathrm{ha}^{-1}$ was obtained with weed free check. Among the weed control treatments pre-emergence application of pendimethalin at $1.0 \mathrm{~kg} \mathrm{ha}{ }^{-1}$ on 3 DAS $f b$ HW on 20DAS + pendimethalin at $1.0 \mathrm{~kg} \mathrm{ha}^{-1}$ on 20 DAS and preemergence application of pendimethalin at $1.0 \mathrm{~kg} \mathrm{ha}^{-1}$ on 3 DAS $f b$ HW on 20 DAS + pendimethalin at $0.5 \mathrm{~kg} \mathrm{ha}^{-1}$ on 20 DAS $\left(\mathrm{T}_{6}\right)$ registered the higher gross return of Rs. 62353 and Rs.57235 ha $^{-1}$. 
Table.1 Effect of weed control treatments on Total weed density $\left(\right.$ No. $\left.\mathrm{m}^{-2}\right)$ and total weed dry weight $\left(\mathrm{kg} \mathrm{ha}^{-1}\right)$ in irrigated blackgram

\begin{tabular}{|c|c|c|c|c|c|c|c|}
\hline \multirow{2}{*}{ T. No } & \multirow{2}{*}{ Treatments } & \multicolumn{3}{|c|}{ Total weed density $\left(\right.$ No.m $\left.^{-2}\right)$} & \multicolumn{3}{|c|}{ Total weed dry weight $\left(\mathrm{kg} \mathrm{ha}^{-1}\right)$} \\
\hline & & 20 DAS & 50 DAS & HARVEST & 20 DAS & 50 DAS & HARVEST \\
\hline $\mathrm{T}_{1}$ & Pendi at $1.0 \mathrm{~kg} \mathrm{ha}^{-1}$ on $3 \mathrm{DAS}$ & $\begin{array}{l}31.43 \\
(3.29)\end{array}$ & $\begin{array}{r}104.49 \\
(5.94)\end{array}$ & $\begin{array}{l}77.14 \\
(5.11)\end{array}$ & $\begin{array}{c}158.70 \\
(7.23)\end{array}$ & $\begin{array}{l}468.51 \\
(12.43)\end{array}$ & $\begin{array}{l}815.06 \\
(16.39)\end{array}$ \\
\hline $\mathrm{T}_{2}$ & Pendi at $1.0 \mathrm{~kg} \mathrm{ha}^{-1}$ on $3 \mathrm{DAS}+$ pendi at $0.5 \mathrm{~kg} \mathrm{ha}^{-1}$ on $20 \mathrm{DAS}$ & $\begin{array}{l}31.16 \\
(3.29)\end{array}$ & $\begin{array}{l}82.71 \\
(5.29)\end{array}$ & $\begin{array}{l}50.60 \\
(4.14)\end{array}$ & $\begin{array}{c}151.80 \\
(7.08)\end{array}$ & $\begin{array}{l}312.17 \\
(10.21)\end{array}$ & $\begin{array}{l}603.10 \\
(14.19)\end{array}$ \\
\hline $\mathrm{T}_{3}$ & Pendi at $1.0 \mathrm{~kg} \mathrm{ha}^{-1}$ on $3 \mathrm{DAS}+$ pendi at $1.0 \mathrm{~kg} \mathrm{ha}^{-1}$ on $20 \mathrm{DAS}$ & $\begin{array}{l}31.67 \\
(3.30)\end{array}$ & $\begin{array}{l}63.73 \\
(4.66)\end{array}$ & $\begin{array}{l}40.03 \\
(3.71)\end{array}$ & $\begin{array}{c}158.86 \\
(7.23)\end{array}$ & $\begin{array}{c}280.34 \\
(9.67)\end{array}$ & $\begin{array}{l}594.59 \\
(14.09)\end{array}$ \\
\hline $\mathrm{T}_{4}$ & $\begin{array}{l}\text { Pendi at } 1.0 \mathrm{~kg} \mathrm{ha}^{-1} \text { on } 3 \mathrm{DAS}+\text { quizolofop-ethyl } 0.050 \mathrm{~kg} \mathrm{ha}^{-1} \text { on } \\
20 \text { DAS }\end{array}$ & $\begin{array}{l}31.84 \\
(3.31)\end{array}$ & $\begin{array}{l}76.37 \\
(4.94)\end{array}$ & $\begin{array}{l}71.17 \\
(4.91)\end{array}$ & $\begin{array}{l}155.02 \\
(7.14)\end{array}$ & $\begin{array}{l}357.93 \\
(10.68)\end{array}$ & $\begin{array}{l}672.63 \\
(14.97)\end{array}$ \\
\hline $\mathrm{T}_{5}$ & Pendi at $1.0 \mathrm{~kg} \mathrm{ha}^{-1}$ on 3 DAS $f b \mathrm{HW}$ on 20 DAS & $\begin{array}{l}32.59 \\
(3.35)\end{array}$ & $\begin{array}{l}78.88 \\
(5.17)\end{array}$ & $\begin{array}{l}58.94 \\
(4.48)\end{array}$ & $\begin{array}{l}148.80 \\
(7.01)\end{array}$ & $\begin{array}{l}307.85 \\
(10.12)\end{array}$ & $\begin{array}{l}652.00 \\
(14.72)\end{array}$ \\
\hline $\mathrm{T}_{6}$ & $\begin{array}{l}\text { Pendi at } 1.0 \mathrm{~kg} \mathrm{ha}^{-1} \text { on } 3 \mathrm{DAS} f b \mathrm{HW} \text { on } 20 \mathrm{DAS}+\text { pendi at } 0.5 \\
\mathrm{~kg} \mathrm{ha}^{-1} \text { on } 20 \mathrm{DAS}\end{array}$ & $\begin{array}{l}34.34 \\
(3.43)\end{array}$ & $\begin{array}{l}58.33 \\
(4.46)\end{array}$ & $\begin{array}{l}38.20 \\
(3.63)\end{array}$ & $\begin{array}{c}139.03 \\
(6.79)\end{array}$ & $\begin{array}{c}252.45 \\
(9.18)\end{array}$ & $\begin{array}{l}470.82 \\
(12.51)\end{array}$ \\
\hline $\mathrm{T}_{7}$ & $\begin{array}{l}\text { Pendi at } 1.0 \mathrm{~kg} \mathrm{ha}^{-1} \text { on } 3 \mathrm{DAS} f b \mathrm{HW} \text { on } 20 \mathrm{DAS}+\text { pendi at } 1.0 \\
\mathrm{~kg} \mathrm{ha}^{-1} \text { on } 20 \mathrm{DAS}\end{array}$ & $\begin{array}{l}33.40 \\
(3.37)\end{array}$ & $\begin{array}{l}44.43 \\
(3.88)\end{array}$ & $\begin{array}{l}34.71 \\
(3.47)\end{array}$ & $\begin{array}{c}147.52 \\
(6.98)\end{array}$ & $\begin{array}{c}198.00 \\
(8.15)\end{array}$ & $\begin{array}{l}453.07 \\
(12.23)\end{array}$ \\
\hline $\mathrm{T}_{8}$ & Hand weeding twice at 20 and 40 DAS & $\begin{array}{l}90.35 \\
(5.45)\end{array}$ & $\begin{array}{l}35.79 \\
(3.48)\end{array}$ & $\begin{array}{l}64.70 \\
(4.67)\end{array}$ & $\begin{array}{r}225.49 \\
(8.60)\end{array}$ & $\begin{array}{c}210.00 \\
(9.51)\end{array}$ & $\begin{array}{l}593.00 \\
(13.86)\end{array}$ \\
\hline $\mathrm{T}_{9}$ & Weed free check & $\begin{array}{c}0.00 \\
(0.71)\end{array}$ & $\begin{array}{c}0.00 \\
(0.71)\end{array}$ & $\begin{array}{c}0.00 \\
(0.71)\end{array}$ & $\begin{array}{c}0.00 \\
(0.71)\end{array}$ & $\begin{array}{c}0.00 \\
(0.71)\end{array}$ & $\begin{array}{c}0.00 \\
(0.71)\end{array}$ \\
\hline $\mathrm{T}_{10}$ & Unweeded check & $\begin{array}{l}98.42 \\
(5.69)\end{array}$ & $\begin{array}{c}210.03 \\
(8.30)\end{array}$ & $\begin{array}{c}271.00 \\
(9.42)\end{array}$ & $\begin{array}{c}237.43 \\
(8.84)\end{array}$ & $\begin{array}{c}1204.26 \\
(20.04)\end{array}$ & $\begin{array}{l}2035.85 \\
(26.00)\end{array}$ \\
\hline & SEd & 0.39 & 0.44 & 0.38 & 0.43 & 1.00 & 0.84 \\
\hline & $\mathrm{CD}(\mathrm{P}=0.05)$ & 0.82 & 0.94 & 0.80 & 0.90 & 2.11 & 1.76 \\
\hline
\end{tabular}

Data were subjected to $\sqrt{(X+0.5)}$ transformation. Figures in parenthesis are means of transformed values

Pendi- Pendimethalin, DAS- Days after sowing, HW- Hand weeding, $f b$ - Followed by. 
Table.2 Effect of weed control treatments on yield attributes and economics in irrigated blackgram

\begin{tabular}{|c|c|c|c|c|c|c|c|}
\hline \multirow[b]{2}{*}{ T. No } & \multirow[b]{2}{*}{ Treatments } & \multicolumn{3}{|c|}{ Yield attributes } & \multicolumn{3}{|c|}{ Economics } \\
\hline & & $\begin{array}{c}\text { Grain } \\
\text { yield } \\
\left(\mathbf{k g ~ h a}^{1}\right)\end{array}$ & $\begin{array}{c}\text { Haulm } \\
\text { yield } \\
\left(\mathrm{kg} \mathrm{ha}^{-1}\right)\end{array}$ & $\begin{array}{c}\text { Harvest } \\
\text { Index }\end{array}$ & $\begin{array}{c}\text { Gross } \\
\text { returns } \\
\left(\text { Rs ha }^{-1}\right)\end{array}$ & $\begin{array}{l}\text { Net returns } \\
\left(\text { Rs ha }^{-1}\right)\end{array}$ & $\mathrm{B}: \mathrm{C}$ ratio \\
\hline $\mathrm{T}_{1}$ & Pendi at $1.0 \mathrm{~kg} \mathrm{ha}^{-1}$ on $3 \mathrm{DAS}$ & 766 & 1367 & 0.36 & 44177 & 27123 & 2.59 \\
\hline $\mathrm{T}_{2}$ & Pendi at $1.0 \mathrm{~kg} \mathrm{ha}^{-1}$ on $3 \mathrm{DAS}+$ pendi at $0.5 \mathrm{~kg} \mathrm{ha}^{-1}$ on $20 \mathrm{DAS}$ & 869 & 1438 & 0.38 & 49945 & 31510 & 2.71 \\
\hline $\mathrm{T}_{3}$ & Pendi at $1.0 \mathrm{~kg} \mathrm{ha}^{-1}$ on $3 \mathrm{DAS}+$ pendi at $1.0 \mathrm{~kg} \mathrm{ha}^{-1}$ on $20 \mathrm{DAS}$ & 924 & 1475 & 0.39 & 53040 & 33723 & 2.75 \\
\hline $\mathrm{T}_{4}$ & $\begin{array}{l}\text { Pendi at } 1.0 \mathrm{~kg} \mathrm{ha}^{-1} \text { on } 3 \mathrm{DAS}+\text { quizolofop-ethyl } 0.050 \mathrm{~kg} \mathrm{ha}^{-1} \\
\text { on } 20 \mathrm{DAS}\end{array}$ & 782 & 1386 & 0.36 & 45085 & 25649 & 2.32 \\
\hline $\mathrm{T}_{5}$ & Pendi at $1.0 \mathrm{~kg} \mathrm{ha}^{-1}$ on $3 \mathrm{DAS} f b \mathrm{HW}$ on 20 DAS & 816 & 1373 & 0.37 & 46938 & 27394 & 2.40 \\
\hline $\mathrm{T}_{6}$ & $\begin{array}{l}\text { Pendi at } 1.0 \mathrm{~kg} \mathrm{ha}^{-1} \text { on } 3 \mathrm{DAS} f b \mathrm{HW} \text { on } 20 \mathrm{DAS}+\text { pendi at } 0.5 \\
\mathrm{~kg} \mathrm{ha}^{-1} \text { on } 20 \mathrm{DAS}\end{array}$ & 995 & 1673 & 0.37 & 57235 & 36310 & 2.74 \\
\hline $\mathrm{T}_{7}$ & $\begin{array}{l}\text { Pendi at } 1.0 \mathrm{~kg} \mathrm{ha}^{-1} \text { on } 3 \mathrm{DAS} f b \mathrm{HW} \text { on } 20 \mathrm{DAS}+\text { pendi at } 1.0 \\
\mathrm{~kg} \mathrm{ha}^{-1} \text { on } 20 \mathrm{DAS}\end{array}$ & 1087 & 1729 & 0.38 & 62353 & 40546 & 2.86 \\
\hline $\mathrm{T}_{8}$ & Hand weeding twice at 20 and 40 DAS & 823 & 1351 & 0.37 & 47275 & 24266 & 2.05 \\
\hline $\mathrm{T}_{9}$ & Weed free check & 1189 & 1803 & 0.40 & 68111 & 39375 & 2.37 \\
\hline $\mathrm{T}_{10}$ & Unweeded check & 492 & 1082 & 0.31 & 28674 & 13883 & 1.94 \\
\hline & SEd & 35.35 & 66.39 & 1.45 & - & - & - \\
\hline & $\mathrm{CD}(\mathrm{P}=0.05)$ & 74.23 & 139.49 & 3.06 & - & - & - \\
\hline
\end{tabular}

Pendi- Pendimethalin, DAS- Days after sowing, HW- Hand weeding, $f b$ - Followed by. 
Pre-emergence application of pendimethalin at $1.0 \mathrm{~kg}$ ha ${ }^{1}$ on 3 DAS $f b \mathrm{HW}$ on 20 DAS + pendimethalin at $1.0 \mathrm{~kg}$ $\mathrm{ha}^{-1}$ on 20 DAS registered the maximum net return of Rs. $40546 \mathrm{ha}^{-1}$. The highest B: C ratio of 2.86 was registered in pre-emergence application of pendimethalin at $1.0 \mathrm{~kg}$ $\mathrm{ha}^{-1}$ on 3 DAS $f b \mathrm{HW}$ on 20 DAS + pendimethalin at 1.0 $\mathrm{kg} \mathrm{ha}^{-1}$ on 20 DAS.Unweeded check recorded the lowest gross returns of Rs. $28674 \mathrm{ha}^{-1}$ and B:C ratio of 1.94.From these study it could be concluded that preemergence application of pendimethalin at $1.0 \mathrm{~kg} \mathrm{ha}^{-1}$ on 3 DAS $f b$ HW on 20 DAS + pendimethalin at $1.0 \mathrm{~kg} \mathrm{ha}^{-1}$ on 20 DAS is cost effective in controlling the weeds of irrigated blackgram.

From the study, it could be concluded that under irrigated blackgram, the lay by method of pre-emergence application of pendimethalin at $1.0 \mathrm{~kg} \mathrm{ha}^{-1}$ on three days after sowing followed by hand weeding on 20 DAS and sand mix application of pendimethalin at $1.0 \mathrm{~kg} \mathrm{ha}^{-1}$ immediately after hand weeding provided season long weed free condition resulted in higher grain yield, net income and return per rupee invested.

\section{References}

Dixit, J., Harvendra Singh and S.K.S. Bhadauria. 2011. Quizalofop ethyl an effective post emergence to control grassy weeds of groundnut. Ann. Pl. Soil Res., 14(1): 22-24.

Dungarwal, H.S., P.C. Chaplot and B.L. Nagda. 2003. Chemical weed control in mungbean (Phaseolus radiates L.). Indian J. Weed Sci., 35: 283-284.

Kalaiselvi, C., C. Loganathan, P. Parameswaran and O.S. Kandasamy. 1998. Effect of weed and nutrient management on yield attributes and yield in groundnut based intercropping system. Pestology, 22 (3): 12-16.

Pramanik, S.C., 2009. Rainwater management techniques for successful production of pulses in rainfed areas. Indian fmg, 58(12): 15-18.

\section{How to cite this article:}

Agila C. and Chinnamuthu C. R. 2017. Yield and Economics of Irrigated Blackgram under Lay by Method of PreEmergence Herbicide Application. Int.J.Curr.Res.Aca.Rev. 5(8), 25-29.

doi: https://doi.org/10.20546/ijcrar.2017.508.004 